\title{
Skin and maxillary sinus involvement of colon cancer
}

\author{
Murat Akyol ${ }^{1}$, Lutfiye Demir ${ }^{1}$, Ahmet Dirican ${ }^{1}$, Nese Ekinci², Alper Can ${ }^{1}$, Vedat Bayoglu' ${ }^{1}$, Ahmet Alacacioglu ${ }^{1}$, \\ Yuksel Kucukzeybek ${ }^{1}$, Suna Cokmert ${ }^{1}$, Cigdem Erten ${ }^{1}$, Fulya Cakalagaoglu², Mustafa Oktay Tarhan ${ }^{1}$ \\ ${ }^{1}$ Izmir Katip Celebi University, Ataturk Training and Research Hospital, Department of Medical Oncology \\ IIzmir Katip Celebi University, Ataturk Training and Research Hospital, Department of Pathology
}

Contemp Oncol (Pozn) 2014; 18 (1): 70-72 DOI: $10.5114 /$ wo.2014.40457

Cutaneous and maxillary sinus metastases of malignancies are not frequent. The most common malignancies metastasizing to the skin in females are breast cancer (69\%), colorectal cancer (9\%), malignant melanoma (5\%) and ovarian cancer (4\%). However, lung cancer (24\%) followed by colorectal cancer (19\%), malignant melanoma $(13 \%)$ and cancer of the oral cavity (12\%) are the common sites in males [1, 2]. Herein, we present a colon cancer patient with maxillary sinus and skin involvement, which are rarely seen localizations of colon cancer metastasis.

A 62-year-old male patient underwent a surgical operation of right hemicolectomy and was diagnosed with stage III colon adenocarcinoma (pT3N1M0) in May, 2006. The pathology was compatible with moderately differentiated adenocarcinoma (Fig. 1) with clear surgical margins but with vascular and neural invasion. Computed tomography (CT) of the thorax and abdomen revealed no metastatic lesions and he received adjuvant oxaliplatin-5FU based chemotherapy for 12 cycles (Nordic-FLOX regimen; oxaliplatin $85 \mathrm{mg} / \mathrm{m}^{2}$ day $1,5 \mathrm{FU} 500 \mathrm{mg} / \mathrm{m}^{2}$ and calcium folinate $60 \mathrm{mg} / \mathrm{m}^{2}$ day 1 and 2 in each 14-day cycle). However, he locally relapsed in September 2011 and underwent surgery for the second time due to intestinal obstruction. Local recurrence at the anastomosis was observed and it was surgically resected with negative surgical margins. Postoperative CT scan revealed multiple lung metastases;

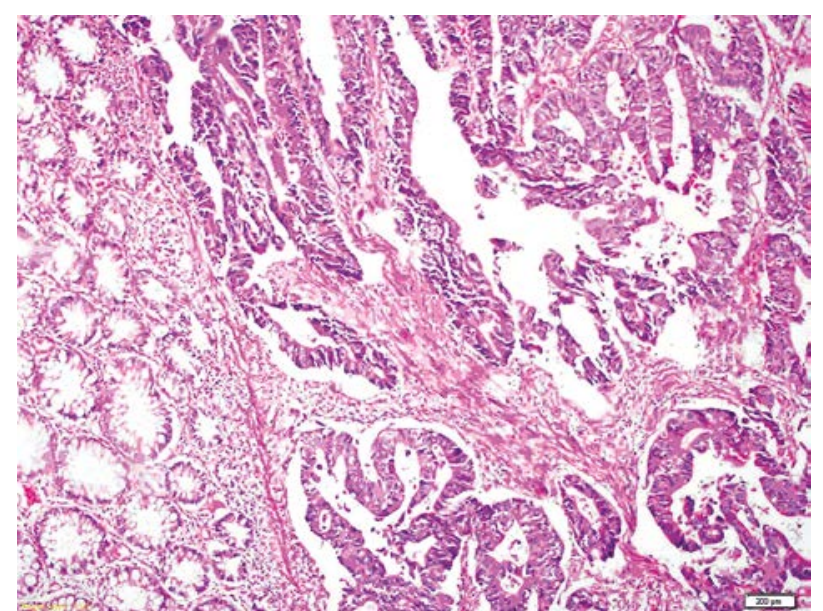

Fig. 1. Moderately differentiated colonic adenocarcinoma with hematoxylin-eosin staining (magnification 40x) thus, a combination chemotherapy of CapeOX-bevacizumab (capecitabine: $2000 \mathrm{mg} / \mathrm{m}^{2}$ for 14 days, oxaliplatin $130 \mathrm{mg} / \mathrm{m}^{2}$ day 1 and bevacizumab $7.5 \mathrm{mg} / \mathrm{kg}$ day 1 in each 21-day cycle) was started in November, 2011. After three cycles, newly appearing skin metastases with nodular pattern were observed on lips, and the skin of the nape and face with a swelling on the maxillary region of the face (Fig. 2A, B). Serum CEA and CA19-9 level at that time were in normal ranges. Magnetic resonance imaging (MRI) of the maxillary sinus demonstrated a $36 \mathrm{~mm} \times 46$ $\mathrm{mm} \times 43 \mathrm{~mm}$-sized infiltrative mass on the anterior wall of the maxillary sinus with orbital invasion (Fig. 3A). The biopsy taken from one of the skin lesions was compatible with metastasis of adenocarcinoma of colon origin (Fig. 3B). Immunohistochemical staining was positive for CK2O (cytokeratin 20) and negative for CK7 (cytokeratin 7) (Fig. 4). Together with skin metastases, also CTscan revealed radiological progression in lung metastases. After then, a combination chemotherapy including irinotecan and capecitabine (irinotecan $180 \mathrm{mg} / \mathrm{m} 2 \mathrm{dl}$, capecitabine 1000 $\mathrm{mg} / \mathrm{m}^{2} 14$ days in each 21-day cycle) was started as a second-line regimen. After two cycles, the skin and maxillary sinus metastases progressed and a cetuximab-irinotecan combination (cetuximab $400 \mathrm{mg} / \mathrm{m}^{2}$ for initial dose and then $250 \mathrm{mg} / \mathrm{m}^{2}$ each week, irinotecan $180 \mathrm{mg} / \mathrm{m}^{2}$ dl every 2 weeks) was started as third-line therapy. He received the second cycle of this regimen last week and he is still alive after six months from the diagnosis of skin and maxillary sinus metastases.

Cutaneous metastases of malignancies are not frequent. Distant cutaneous metastasis occurs by vessel invasion and hematogenous spreading while lymphatic invasion and direct spreading are the most common ways for local cutaneous recurrence $[3,4]$. The most common sites of skin metastases are the pelvic region, upper extremities, chest, head and neck region and rarely the glans penis in men [4]. The largest case series of skin metastases from colon cancer in the literature included 80 patients; in 77 cases they were located on the skin of the abdomen [5].

Colorectal cancer is the third most commonly diagnosed cancer in males and the second in women [6]. The most common metastatic sites for colorectal cancer are liver and lung respectively [7]. Skin metastases due to colorectal cancer account for only $5 \%$ of metastatic skin 

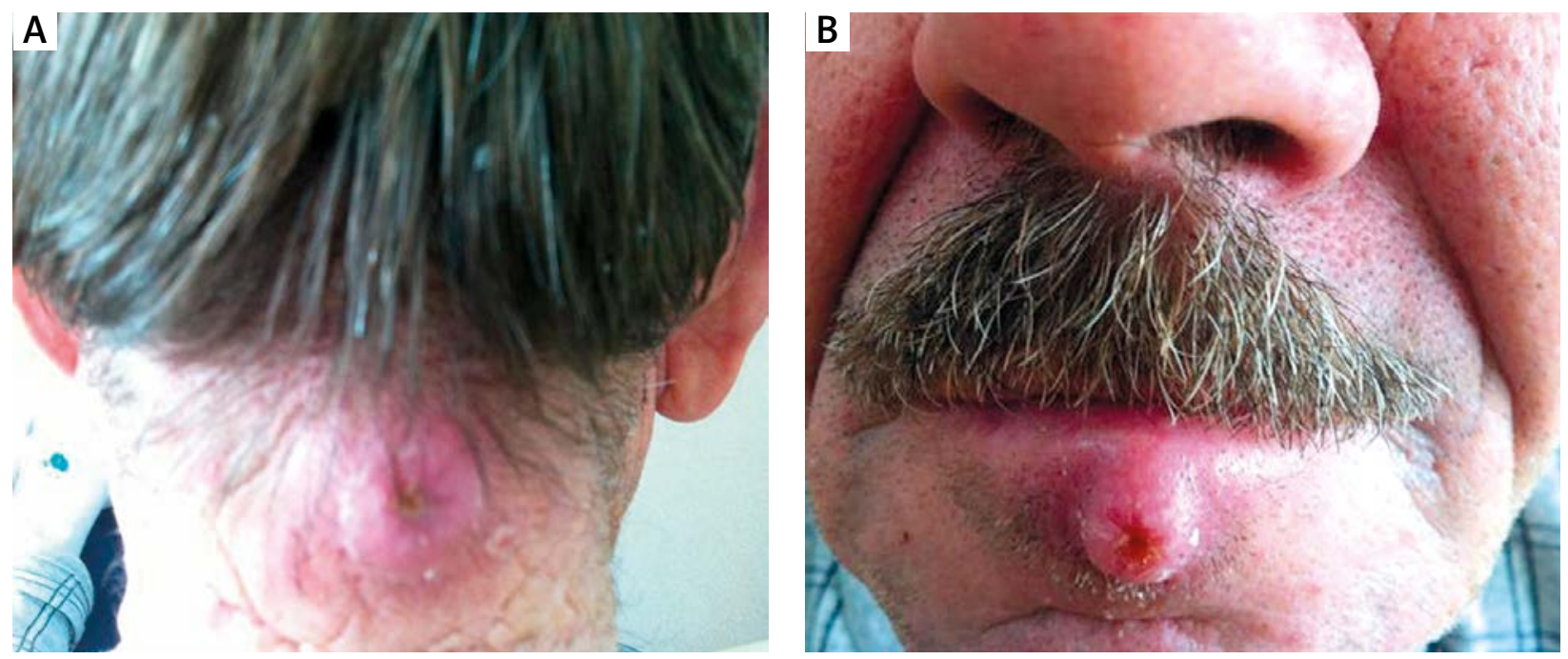

Fig. 2A, B. Photographs of the patient with involvement of the skin of lips and nape

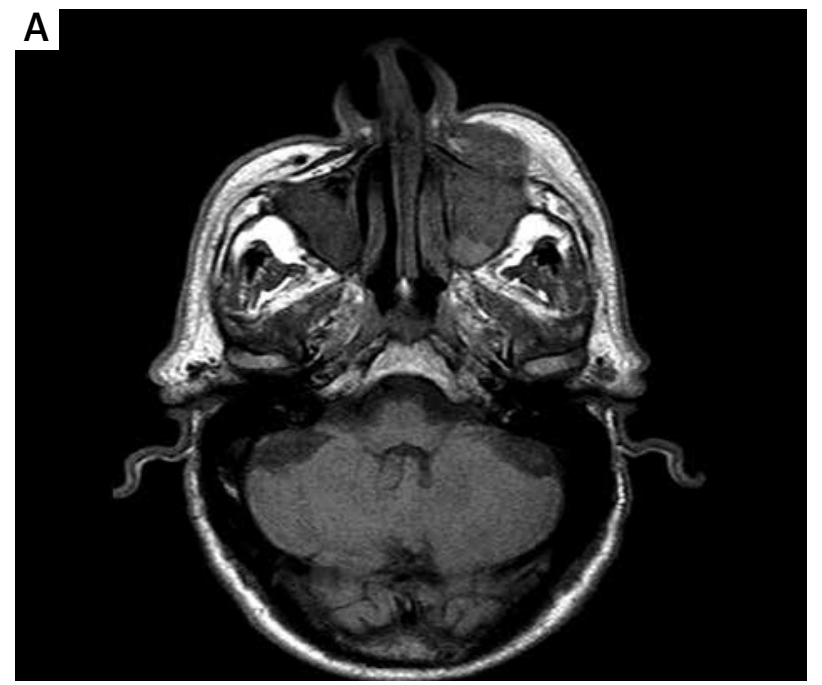

Fig. 3A. Involvement of maxillary sinus with adenocarcinoma in the magnetic resonance imaging

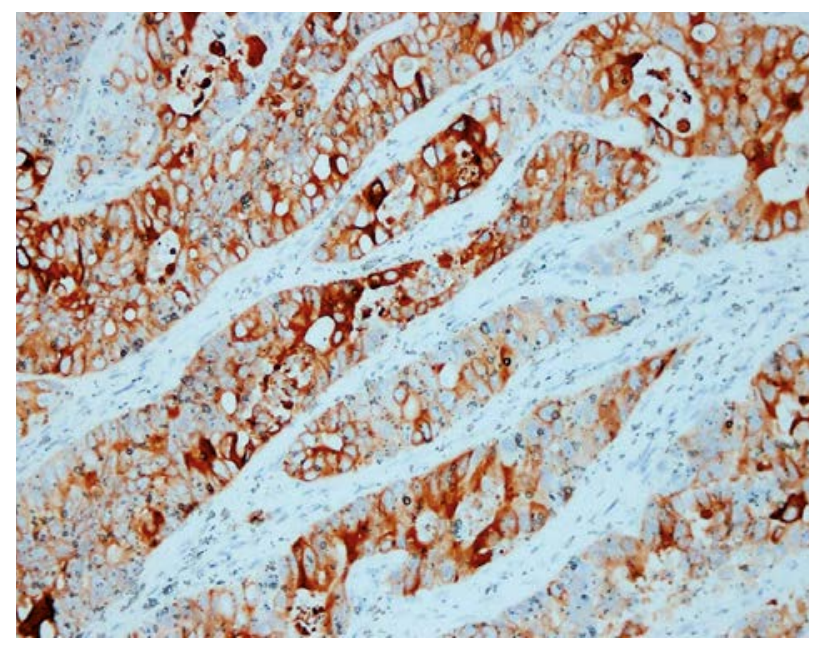

Fig. 4. Immunohistochemically positive staining with CK2O as seen in brown color (magnification 40x)

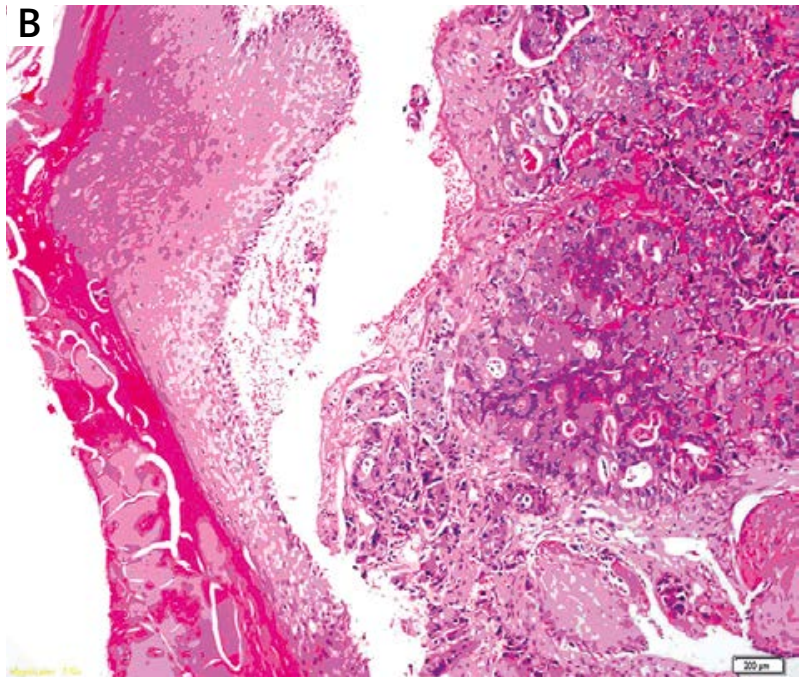

Fig. 3B. Image of adenocarcinoma focus under the normal squamous epithelium of the skin with hematoxylin-eosin staining $(\times 40$ magnification)

cancers [8], and usually are located on the skin of the abdomen followed by extremities, glans penis in men and head-neck region [9-12]. The presentation of skin metastases can be nodular, ulcerative, or as small tumor deposits [13]. Lookingbill et al. reviewed 4020 patients with metastatic cancer, of whom 413 had colorectal carcinoma, and only 18 (4.4\%) patients were determined to have cutaneous metastases; of those only one patient had metastatic facial involvement [14]. Cutaneous metastases usually occur approximately 3 years after diagnosis and median overall survival time from the diagnosis of skin metastasis is reported as 18-20 months [4]. The survival of a patient with colon cancer with scalp metastasis was reported as only a few months in a recent paper [8].

Malignancies of the paranasal sinuses are also extremely rare; they account for around 3-5\% of all head-and-neck cancers and less than $1 \%$ of all malignancies $[15,16]$. Maxillary sinus is the most common reported involvement site 
of paranasal sinus malignancies. Ten to $20 \%$ of all sinonasal malignancies are in adenocarcinoma histology [17]. Many of these originate from salivary glands; however, some of them have similar histologic features as colonic adenocarcinoma and thus are named as "intestinal type adenocarcinoma". The distinction between the metastasis of colonic adenocarcinoma and primary adenocarcinoma of paranasal sinuses is generally performed by using immunohistochemical features of the tumor. CK20-positive/ CK7-negative immunoprofile is considered specific for colorectal tumors. The prognosis of metastatic tumors of paranasal sinuses is also reported to be poor [18]. Because of its rarity, only a few cases with poor prognosis are reported in the literature [19-21].

In this case, the patient had skin metastases in the head and neck region and in the pelvic region, which were previously reported as rare localizations of skin metastases in the literature. Additionally he had maxillary sinus involvement at the same time as skin metastases. Our patient developed nodular skin and maxillary sinus metastases 5 years after the diagnosis of colon cancer. The patient did not respond to the first two lines of chemotherapy and progressed rapidly in five months. He is still being treated with third line chemotherapy. In conclusion, skin and maxillary sinus involvement in patients with colorectal cancer rarely occurs and predicts poor prognosis, as was the case in our patient.

\section{References}

1. Aasi SZ, Leffell DJ. Cancer of the skin. In: DeVita VT, Heilman S, Rosenberg SA. Cancer: principles and practise of oncology. 7th ed. Lippincott Williams \& Wilkins, Philadelphia 2005; p. 1717-4.

2. Sarid D, Wigler N, Gutkin Z, Merimsky O, Leider-Trejo L, Ron IG. Cutaneous and subcutaneous metastases of rectal cancer. Int J Clin Oncol 2004; 9: 202-5.

3. Brownstein MH, Helwig EB. Patterns of cutaneous metastasis. Arch Dermatol 1972; 105: 862-8.

4. Attili VS, Rama Chandra C, Dadhich HK, Sahoo TP, Anupama G, Bapsy PP. Unusual metastasis in colorectal cancer. Indian J Cancer 2006; 43: 93-5.

5. Saeed S, Keehn CA, Morgan MB. Cutaneous metastasis: a clinical, pathological, and immunohistochemical appraisal. J Cutan Pathol 2004; 31: 419-30.

6. Jemal A, Bray F, Center MM, Ferlay J, Ward E, Forman D. Global cancer statistics. Cancer J Clin 2011; 61: 69-90.

7. August DA, Ottow RT, Sugarbaker PH. Clinical perspective of human colorectal cancer metastasis. Cancer Metastasis Rev 1984; 3 : 303-24.

8. Horiuchi A, Nozawa K, Akahane T, et al. Skin metastasis from sigmoid colon cancer. Int Surg 2011; 96: 135-8.

9. Proffer LH, Czarnik KL, Sartori CR. Colon carcinoma cutis: a case report. Cutis 1999; 63: 301-2.

10. Camci C, Türk HM, Büyükberber S, Karakök M, Koruk M, Beyazity Y, Inalöz HS. Colon carcinoma with synchronous subcutaneous and osseous metastasis: a case report. J Dermatol 2002; 29: 362-5.

11. Kilickap S, Aksoy S, Dinçer M, Saglam EA, Yalçin S. Cutaneous metastases of signet cell carcinoma of the rectum without accompanying visceral involvement. South Med J 2006; 99: 1137-9.

12. Torzilli G, Cremascoli G, Cattaneo S, Stefanini P, Olivari N. Drainsite tumour recurrence after laparotomy resection for colorectal cancer. Eur I Surg Oncol 1999; 25: 546-7.

13. Brownstein MH, Helwig EB. Spread of tumors to the skin. Arch Dermatol 1973; 107: 80-6.
14. Lookingbill DP, Spangler N, Helm KF. Cutaneous metastases in patients with metastatic carcinoma: a retrospective study of 4020 patients. J Am Acad Dermatol 1993; 29: 228-36.

15. Roush GC. Epidemiology of cancer of the nose and paranasal sinuses: current concepts. Head Neck Surg 1979; 2: 3-11.

16. Ayiomamitis A, Parker L, Havas T. The epidemiology of malignancies of the nasal cavities, paranasal sinuses and middle ear in Canada. Arch Otorhinolaryngol 1988; 244: 367-71.

17. Bernstein JM, Montgomery WW, Balogh K Jr. Metastatic tumors to the maxilla, nose and paranasal sinuses. Laryngoscope 1966; 76: 621-50

18. Mickel RA, Zimmerman MC. The sphenoid sinus - a site for metastasis. Otolaryngol Head Neck Surg 1990; 102: 709-16.

19. Cama E, Agostino S, Ricci R, Scarano E. A rare case of metastases to the maxillary sinus from sigmoid colon adenocarcinoma. ORL J Otorhinolaryngol Relat Spec 2002; 64: 364-7.

20. bin Sabir Husin Athar PP, bte Ahmad Norhan N, bin Saim L, bin Md Rose I, bte Ramli R. Metastasis to the sinonasal tract from sigmoid colon adenocarcinoma. Ann Acad Med Singapore 2008; 37: 788-3.

21. Somali I, Yersal O, Kilçiksiz S. Infratemporal fossa and maxillary sinus metastases from colorectal cancer: a case report. J BUON 2006; 11: 363-5.

\section{Address for correspondence}

\section{Lutfiye Demir, MD}

Basin Sitesi, Karabaglar, Izmir-Turkey tel. +90 23224444 44/2923,

fax +90232 2431530

e-mail: demir.lutfiye77@gmail.com

Submitted: 19.07 .2012

Accepted: $\quad$ 2.10.2012 